\title{
A metric driving local action for global biodiversity conservation
}

\section{Axel Rossberg ( $\nabla$ a.rossberg@qmul.ac.uk)}

Queen Mary University of London

Jacob O'Sullivan

Queen Mary University of London

Christopher Terry

Queen Mary University of London

Nadav Shnerb

Bar-llan University

Article

Keywords:

Posted Date: February 11th, 2022

DOI: https://doi.org/10.21203/rs.3.rs-1291460/v1

License: (9) This work is licensed under a Creative Commons Attribution 4.0 International License.

Read Full License 


\section{A metric driving local action for global biodiversity}

\section{conservation}

January 24, 2022

Axel G. Rossberg ${ }^{1}$, Jacob D. O’Sullivan ${ }^{1}$, J. Christopher D. Terry ${ }^{1}$ and Nadav M. Shnerb ${ }^{2}$

$6{ }^{1}$ School of Biological and Chemical Sciences, Queen Mary University of London, Mile End Road, London,

7 E1 4NS, United Kingdom

$8 \quad 2$ Department of Physics, Bar-Ilan University, Ramat-Gan IL52900, Israel

$9 \quad$ Corresponding author: Axel G. Rossberg, School of Biological and Behavioural Sciences, Queen

10 Mary University of London, Mile End Road, London E1 4NS, United Kingdom, (a.rossberg@qmul.ac.uk, $11 \quad+447551396243)$ 


\begin{abstract}
Difficulties identifying appropriate biodiversity impact metrics remain a major barrier to inclusion of biodiversity considerations in environmentally responsible investment. We propose and analyse a simple science-based local metric: the sum of the proportions that all local populations contribute to their global species abundances, with a correction for species close to extinction. It links mathematically to a widely used global biodiversity indicator, the Living Planet Index, for which we propose an improved formula that directly addresses the known problem of singularities caused by extinctions. We show that, in an ideal market, trade in our metric would lead to near-optimal conservation resource allocation, emphasising support of extinction-threatened species. Barriers to adoption are low, as use of the metric does not require an institutional certification system. Used in conjunction with other metrics, potential areas of application include biodiversity related financial disclosures and voluntary or legislated no net biodiversity loss policies.
\end{abstract}

\title{
Main
}

The rapid recent growth of markets for responsible investments considering Environmental, Social and Governance (ESG) concerns ${ }^{1}$, and the growing attention biodiversity receives in this context ${ }^{213}$, highlight the need for metrics of biodiversity impacts for use by businesses and in financial markets 2 2415.

Requirements on such metrics are different from those for metrics used in the policy context, where some flexibility is desired and a balanced representation of multiple objectives sought ${ }^{6}$. In the business context, metric simplicity is of the essence. Non-expert market participants must understand the impacts their decisions have on a metric ${ }^{4}$. Metric definitions should be rigorous and robust enough to withstand strong financial incentives to game them ${ }^{4}$. Metric values should be comparable across a wide range of business activities ${ }^{7}$. Importantly, biodiversity metrics should support a simple narrative about biodiversity 
conservation in communication between business, customers, and the general public ${ }^{4}$. To reduce exposure of market participants to risks from redefinition of metrics as flaws become apparent ${ }^{8}$, metrics should be 'science based' in the sense that all choices made in their definition have a scientific rationale. Metrics should be consistent across the spatial scales on which business activity occurs. Ideally, they should also be marketable and the resulting market forces trigger local action that adds up to protection and recovery of global biodiversity.

The Taskforce on Nature-related Financial Disclosures (TNFD), which aims to develop by 2023 a framework, suitable for the financial sector, "for organisations to report and act on evolving nature-related risks" 9 , builds on metric selection criteria developed by UNEP-WCMC's Aligning Biodiversity Measures for Business $5[10$, however, with a stronger view on comparability within and across business sectors. These emphasise relevance, transparency, consistency and rigour of metrics, alongside the need for completeness of the metric set.

Here we propose a new metric, 'Biodiversity Stewardship Credits' (BSC), designed to address this need with regards to the protection of biodiversity for its 'intrinsic value' and as a matter of intergenerational justice. Specifically, it is designed to link local action mathematically to its impact on the Living Planet Index (LPI) $\frac{1112}{}$, one of the most widely cited global diversity indicators currently in use.

To overcome technical and conceptual problems arising when populations of species entering the LPI approach zero 1213 , we begin by introducing a variant of this indicator that avoids these problems. For this variant we demonstrate that, under certain simplifying assumptions, it quantifies long-term extinction risk, thus providing a solid scientific basis for the marketable metric (BSC) that we subsequently derive.

\section{Extinction risk and regularisation of the Living Plant Index}

The LPI quantifies the change in the geometric mean of the abundances of species in a given taxonomic or functional group (below: group of species), relative to a baseline year 12 . Geometric mean abundance 
biodiversity indices of this type stand out by their combined simplicity, favourable statistical properties $\frac{14}{\text {, }}$ intuitive accessibility and ecological plausibility 14 . Not all species are regularly surveyed at sufficient accuracy to compute the LPI directly, but the index can be estimated as an appropriately weighted geometric average from existing survey time series $\frac{12}{2}$. These estimates are now regularly determined and published under the leadership of the World Wildlife Fund 11 . The LPI is amongst the most frequently cited biodiversity status indicators in the media, evidencing its wide acceptance by policy makers and the public. It can be interpreted as the typical amount by which species abundances have increased or decreased since the baseline. Because geometric mean abundance is highly sensitive to changes in the abundances of rare species $\frac{13}{13}$, LPI is sensitive to pressures on rare species even when the total biomass of the group of species represented changes only little.

Mathematically, the LPI is often studied in terms of the sum $\mathcal{L}$ of the natural logarithms (notation ' $\log x$ ') of the global abundances $N_{i}$ of all $S$ species $i$ in the group of interest:

$$
\mathcal{L}=\sum_{i}^{S} \log N_{i}
$$

In place of abundance $N_{i}$, other measures of population size can be used, such as population biomass or area covered, whichever approximates total reproductive value ${ }^{15}$ well.

If we denote by $\mathcal{L}_{0}$ the value of $\mathcal{L}$ in the baseline year, current LPI can be computed from $\mathcal{L}$ as

$$
\mathrm{LPI}=\exp \left(\frac{\mathcal{L}-\mathcal{L}_{0}}{S}\right)
$$

${ }_{3}$ If one of the $S$ species went extinct since the baseline year, $\mathcal{L}$ attains a value of negative infinity and LPI becomes zero, irrespective of all other species. Practical calculations of the LPI avoid this mathematical singularity by introducing ad hoc rules to handle rare or extinct species ${ }^{16}$. Satisfactory resolution of this issue is difficult because the interpretation of geometric mean abundance metrics in terms of ecological first 
principles remains incomplete. In the following we propose such an interpretation that naturally overcomes the singularity.

It has been shown on long-term time series data, e.g., for trees $\frac{1718}{}$ fish $^{18}$, herbaceous plants $\frac{18}{}$, and $\operatorname{birds}^{17}$ that the populations of most species exhibit random walks on the $\log (N)$ axis with mean-squared increments that are largely independent of $N$, a phenomenon known as 'environmental stochasticity' (caused by variability in both the abiotic environment and other, co-occurring species). For a given population, we denote the mean square of this increment during a time interval of length $\Delta t$, divided $\Delta t$, by $v_{\mathrm{e}}$. These studies also reveals little if any detectable drift towards smaller population sizes or any preferred value.

On top of environmental stochasticity, the discrete nature of life-history events (e.g. birth, germination, death etc.) generates additional variability in population sizes, known as 'demographic stochasticity', for which mean-squared increments in $N$ over a given time interval $\Delta t$ scale proportionally to $N$. We denote the corresponding proportionality constant, divided by $\Delta t$, by $v_{\mathrm{d}}$. Demographic stochasticity is widely understood to be negligible compared to environmental stochasticity, except for small population sizes 19 . The ratio $N^{*}=v_{\mathrm{d}} / v_{\mathrm{e}}$ specifies the population size below which demographic stochasticity dominates over environmental stochasticity.

In Box 1 we show for populations driven by environmental and demographic stochasticity that the quantity

$$
\mathcal{L}_{\text {reg }}=\sum_{i}^{S} \log \left(1+N_{i} / N_{i}^{*}\right)
$$

is, for a given group of $S$ species, proportional to the expected number of surviving species after a long time 5 . In view of this intuitive ecological interpretation, we propose use of the regularised quantity $\mathcal{L}_{\text {reg }}$ in place of $\mathcal{L}$ and correspondingly define the regularised Living Planet Index as

$$
\mathrm{LPI}_{\mathrm{reg}}=\exp \left(\frac{\mathcal{L}_{\mathrm{reg}}-\mathcal{L}_{\mathrm{reg}, 0}}{S}\right)
$$


Box 1 The regularised Living Planet Index as a measure for extinction risk

We show that with a small modification the LPI can be understood as quantifying long-term extinction risk. We model the change in the population size of a species over a time interval $\Delta t$ as

$$
N(t+\Delta t)=\exp \left[\xi(t) \sqrt{v_{\mathrm{e}} \Delta t}\right] N(t)+\xi^{\prime}(t) \sqrt{v_{\mathrm{d}} \Delta t} N(t)
$$

where $\xi(t)$ and $\xi^{\prime}(t)$ denote independent standard normal random numbers. Parameters $v_{\mathrm{e}}$ and $v_{\mathrm{d}}$ represent the strengths of environmental and demographic stochasticity, respectively. Formally taking the limit $\Delta t \rightarrow 0$, standard procedures lead to an approximation of this process by the Ito stochastic differential equation

$$
d N=\frac{v_{\mathrm{e}}}{2} N d t+\sqrt{v_{\mathrm{d}} N+v_{\mathrm{e}} N^{2}} d W_{t}
$$

where $W_{t}$ represents a Wiener process (Brownian motion). The first term on the right-hand-side describes drift to larger values. It goes back to the fact that the expectation value of the log-normal distribution $\exp \left[\xi(t) \sqrt{v_{\mathrm{e}} \Delta t}\right]$ in Eq. [3] is $\exp \left(v_{\mathrm{e}} \Delta t / 2\right)$. If one were to formulate this process in terms of $\log (N)$ rather then $N$, this term would disappear. However, demographic stochasticity would then generate another drift term instead. To eliminate drift altogether, we express population sizes in terms of $x=\log \left(1+N / N^{*}\right)$, where $N^{*}=v_{\mathrm{d}} / v_{\mathrm{e}}$ is the population size at which environmental and demographic stochasticity have the same strength (see Eq. 4). Note that $x$ becomes zero when a species goes extinct $(N=0)$. Applying Ito's lemma, this change of variables simplifies Eq. 4 to

$$
d x=\sqrt{\left(1-e^{-x}\right) v_{\mathrm{e}}} d W_{t}
$$

That is, $x$ performs a Brownian motion, represented by $d x=\sqrt{v_{\mathrm{e}}} d W_{t}$, except when $x$ is of the order of one or smaller.

For $x$ approaching zero from above, the factor $1-e^{-x}$ reduces the magnitude of fluctuations in $x$, slowing down the random walk. As a result, $x$ can get trapped in the region of low $x$, and the vicinity of 0 acts similar to an absorbing boundary 20/21. This effect is reinforced by the breakdown of the diffusion approximation underlying Eqs. (4) and 5 for small $x^{22}$. In reality $N$ and so $x$ reach zero eventually, implying global extinction of that species. We therefore approximate the dynamics of $x$ by simple Brownian motion with an absorbing boundary at $x=023$

Now consider the probability that a species starting from $x=x_{0}$ will still exist after a time $T$, i.e., the probability for $x$ to never reach 0 before $T$. Textbook methods evaluate this to $\operatorname{erf}\left(x_{0} / \sqrt{2 T v_{\mathrm{e}}}\right)$ 24, where erf denotes the error function 25 . For $T$ not too near in the future $\left(T \gg v_{\mathrm{e}}^{-1} x_{0}^{2}\right)$, this simplifies to

$$
\text { (probability of survival until } T)=\sqrt{\frac{2}{\pi T v_{\mathrm{e}}}} x_{0} \text {. }
$$

That is, for any sufficiently large, fixed observation time $T$, the current value of $x_{0}=x=\log \left(1+N / N^{*}\right)$ is directly proportional to the probability of species survival. In Supplementary Information (S1) we demonstrate validity of Eq. 60 for a model with discrete population sizes $N$.

Data suggest that $v_{\mathrm{e}}$ does not usually vary strongly within taxonomic groups 1718. When this is so, $\sum_{i}^{S} \log \left(1+N_{i} / N_{i}^{*}\right)$ is, for a given group of $S$ species, proportional to the expected number of species surviving after a long time $T$.

with $\mathcal{L}_{\text {reg, }, 0}$ denoting the value of $\mathcal{L}_{\text {reg }}$ at the baseline year. These regularised quantities naturally avoid the singularity that arises for extinct species $\left(N_{i}=0\right)$. Yet, LPI and $\mathrm{LPI}_{\mathrm{reg}}$ are nearly identical when all species populations $N_{i}$ are much larger than the corresponding $N_{i}^{*}$.

\section{Quantifying local biodiversity impact}

With global biodiversity quantified in terms of $\mathcal{L}_{\text {reg }}$, the biodiversity impact of any intervention can be quantified as the resulting change $\Delta \mathcal{L}_{\text {reg }}$ in this metric. If the changes in population sizes $\Delta N_{i}$ resulting from a local impact are just small fractions of population sizes $N_{i}$, as will often be the case, $\Delta \mathcal{L}_{\text {reg }}$ is well 
approximated to linear order in $\Delta N_{i}$ as

$$
\Delta \mathcal{L}_{\mathrm{reg}} \approx \sum_{i}^{S} \frac{\partial \mathcal{L}}{\partial N_{i}} \Delta N_{i}=\sum_{i}^{S} \frac{\Delta N_{i}}{N_{i}^{*}+N_{i}}
$$

From Eq. 9 it is clear that $\Delta \mathcal{L}_{\text {reg }}$ weights impacts on globally rare species higher than impacts on common species, thus plausibly providing a measure of pressure on $\left(\Delta \mathcal{L}_{\text {reg }}<0\right)$ or relief to $\left(\Delta \mathcal{L}_{\text {reg }}>0\right)$ global biodiversity.

Any practical determination of $\Delta \mathcal{L}_{\text {reg }}$ would require that the changes in abundances $\Delta N_{i}$ entering Eq. (9) are calculated by comparing measured abundances before and not long after an intervention has occurred. This might raise concerns that the long-term ecological reverberations of an intervention are yet to unfold and so missed by Eq. (9). Since populations are spatially connected through dispersal, local interventions might also impact ecological communities outside the area considered. Indeed, we demonstrated such complex, far-reaching long-term effects of press perturbations in recent simulation of a species-rich metacommunity model 26 . Remarkably, however, these simulations also revealed that, at least in the case of complete eradication of all species on a site (as when building a warehouse in a meadow), the average long-term change in the total population of each species across the metacommunity is nearly identical to the size of the local population removed by the intervention. This suggests that use of Eq. (9) with the short term values of $\Delta N_{i}$ provides a good estimate of the expectation value of the long-term impact, even when ecological complexity makes actual long-term impacts less predictable.

\section{The Biodiversity Stewardship Credit metric}

A disadvantage of using impact metrics in the management of biodiversity is that inaccuracies in the quantification of positive and negative impacts can accumulate, potentially leading to leakage with systematic, unaccounted for biodiversity loss $\frac{7227}{}$. Impact metrics are therefore usefully defined in terms of change in a 
local measures of biodiversity state ${ }^{8}$. However, this approach is not directly applicable here, since LPI and $\mathrm{LPI}_{\text {reg }}$ are difficult to apportion over space. Indices permitting easy spatial disaggregation ${ }^{28}$, on the other hand, tend to have difficulties incentivising the protection of rare species 29 .

As a proposal to overcome this conundrum, we define, for a given area of land (or water), associated Biodiversity Stewardship Credits as

$$
\mathrm{BSC}=\sum_{i}^{S} \frac{n_{i}}{N_{i}^{*}+N_{i}},
$$

with $S, N_{i}$ and $N_{i}^{*}$ defined as above and $n_{i}$ being the local population size of species $i$ that this area sustains (for migratory species scaled by the proportion of time spent in the area).

Comparison of Eqs. (9) and (10) shows that, when all $n_{i}$ are small relative to $N_{i}$, e.g. because the area is small, BSC quantify the short-term impact that would result from removing all species of the group in that area. The metric supports a narrative of biodiversity stewardship because it gives high weight to locally sustained populations of globally rare species.

We note the following four properties of BSC that follow directly from Eq. 10):

1. BSC are additive: the BSC of a combination of non-overlapping areas is simply the sum of their BSC.

2. BSC represent a strictly finite resources: since $n_{i}$ is local and $N_{i}$ global population size, the global sum of all BSC for a given group of species of size $S$ is close to but never larger than $S$.

3. The numerical value of BSC can be interpreted as the effective number of species that the associated area sustains, while accounting for extinction risks through the $N_{i}^{*}$ parameters.

4. While there will always be some uncertainty in measuring BSC, and standards and certification systems help reducing this uncertainty, such external authority is not required. Land holders hold the associated BSC without a need for certification or expert assessment. 
Computation of BSC is simpler than it might first appear. Of those species that occur at a site, just a few will typically dominate the sum in Eq. (10), either because they dominate the local community or because they are globally rare (Box 2). Compared to values of $N_{i}$ larger than about $100-1000, N_{i}^{*}$ is generally sufficiently small that it can be disregarded in the evaluation of Eq. 10 17/19. Usually, BSC can therefore be computed by simply estimating the proportions local populations of the dominant species contribute to their global abundance, and adding up these proportions. Box 2 provides an example. To account for the small, non-dominant contributions from other species, generic statistical estimates can be used.

The largest hurdle in this calculation might be estimation of the global abundances of species. Since nearly all IUCN Red List assessment criteria ${ }^{33}$ involve population size or relevant alternative measures (e.g. number of extant sub-populations), Red List assessments are a valuable source of relevant global abundance data, especially for rare species. Furthermore, a general statistical approach to global abundance estimation has recently been developed and demonstrated for the majority of known birds species ${ }^{30}$. For many other taxonomic and functional groups survey databases with global coverage are now being built, which should permit replication of this result. For land plants, for example, relative global abundances have been estimated from the Botanical Information and Ecology Network (BIEN, http://bien.nceas.ucsb.edu/bien/)

Where required, $N^{*}$ values can be computed as $N^{*}=v_{\mathrm{d}} / v_{\mathrm{e}}$, with $v_{\mathrm{d}}$ and $v_{\mathrm{e}}$ estimated from population time series data as used in calculations of the global LPI $17 \mid 18$, life-history data ${ }^{32[35] 36}$, or combinations thereof 31 .

We are unaware of direct alternatives to BSC that would quantify local contributions to extinction risk. Comparable is the recently proposed Species Threat Abatement and Recovery (STAR) metric ${ }^{44}$. However, STAR is neither a measure of biodiversity state nor has it been designed for marketisation. In Supplementary Information $(\mathrm{S} 2)$ we show that the mathematical structure underlying STAR and BSC is similar, suggesting that these metrics support broadly consistent conservation priorities. 


\section{${ }_{167}$ Incentive structure generated by BSC}

We envision that organisations can usefully include regular BSC accounts in environmental impact reports. The fact that BSC are always positive encourages reporting as complete as logistically possible. The bonuses that environmentally friendly assets currently attract on markets then imply that BSC on their own have monetary value. For example, an asset classification scheme based on BSC per hectare of land held would encourage organisations to acquire high-BSC land and to increase BSC on the land they hold. A market for BSC could emerge as a result. However, it is not a priori clear what the overall effect of the resulting trade in BSC would be. Could it create perverse incentives running contrary to the original objective of biodiversity protection? We study this question in the following, assuming a perfect market.

Let $p$ be the price of one BSC and $C_{\alpha}=C_{\alpha}\left(n_{\alpha, 1}, \ldots, n_{\alpha, S}\right)$ the net present value of the costs of sustaining local population sizes $n_{\alpha, 1}, \ldots, n_{\alpha, S} \geq 0$ in areas of land held by market participant $\alpha$, including costs related to lost opportunities for other uses, minus the net present value of the resulting ecosystem services. The total value of that area is then $V_{\alpha}=-C_{\alpha}\left(n_{1}, \ldots, n_{S}\right)+p \mathrm{BSC}_{\alpha}$ plus value unrelated to $n_{1} \ldots n_{S}$. In a perfect market, market participant $\alpha$ will take measures to maximise $V_{\alpha}$, at which point

$$
0=\frac{\partial V_{\alpha}}{\partial n_{\alpha, i}}=-\frac{\partial C_{\alpha}}{\partial n_{\alpha, i}}+\frac{p}{N_{i}^{*}+N_{i}}-\frac{p n_{\alpha, i}}{\left(N_{i}^{*}+N_{i}\right)^{2}} \quad\left(\text { or } \quad n_{\alpha, i}=0\right)
$$

for all species $1 \leq i \leq S$. The last, negative term in Eq. (11) results because $n_{\alpha, i}$ contributes to total population size $N_{i}\left(\right.$ so $\left.\partial N_{i} / \partial n_{\alpha, i}=1\right)$. The $n_{\alpha, i}=0$ case arises when there are no net benefits at all in sustaining species $i$. In such a case one typically finds that even small populations of $n_{\alpha, i}>0$ do not add value, implying that $\partial C_{\alpha} / \partial n_{\alpha, i}>p /\left(N_{i}^{*}+N_{i}\right)$ at $n_{\alpha, i}=0$

Now, consider the problem of minimising the global costs of sustaining $\mathcal{L}_{\text {reg }}$ at a given level by appropriately choosing the population sizes $n_{\alpha, i} \geq 0$ sustained by each land holder $\alpha$. The solution of this constrained non-linear optimisation problem satisfied the Karush-Kuhn-Tucker conditions $\frac{37}{\sqrt{37}}$ derived from 
the Lagrangian

$$
\sum_{\alpha} C_{\alpha}-\sum_{\alpha, i} \mu_{\alpha, i} n_{\alpha, i}-\lambda \mathcal{L}_{\mathrm{reg}}
$$

189

where $\mu_{\alpha, i}$ and $\lambda$ are the Karush-Kuhn-Tucker multipliers. It leads to the stationarity condition that

$$
-\frac{\partial C_{\alpha}}{\partial n_{\alpha, i}}-\mu_{\alpha, i}+\frac{\lambda}{N_{i}^{*}+N_{i}}=0
$$

for all $\alpha$ and $i$, where either $\mu_{\alpha, i}=0$ and $n_{\alpha, i} \geq 0$ or $\mu_{\alpha, i}>0$ and $n_{\alpha, i}=0$. Comparison of Eqs. (11) and (13) and of the considerations for $n_{\alpha, i}=0$ shows that with $p=\lambda$ the two conditions are identical, except for the term $p n_{\alpha, i} /\left(N_{i}^{*}+N_{i}\right)^{2}$ in Eq. (11). However, this term makes a sizeable contribution compared to the term $p /\left(N_{i}^{*}+N_{i}\right)$ only for when $\alpha$ holds a large proportion of the population of species $i$ (so $n_{\alpha, i}$ and $N_{i}$ are of comparable size). Absent such dominant market participants, an ideal BSC market leads to near optimal allocation of resources to sustain $\mathcal{L}_{\text {reg }}$, and so $\mathrm{LPI}_{\text {reg }}$, at a given level. Larger $\mathrm{LPI}_{\text {reg }}$ correspond to higher BSC prices $p$.

To address the special case of dominant market participants, we now show the following: Firstly, even when rare species are dominantly held by a single market participants, BSC still incentivise protection of these species and growing their populations. Secondly, BSC disincentivise dominance. Consider a market participant whose land is home to the entire global population of some species $i$. The participant's BSC associated with species $i$ are then

$$
\operatorname{bsc}_{i}=\frac{n_{i}}{N_{i}^{*}+n_{i}}
$$

A local population size of $n_{i}=N_{i}^{*}$, for example, earns this market participant 0.5 BSC (Fig. 1). This is plenty and worth preserving, considering that populations of size $N_{i}^{*}$ tend to be comparatively small and 


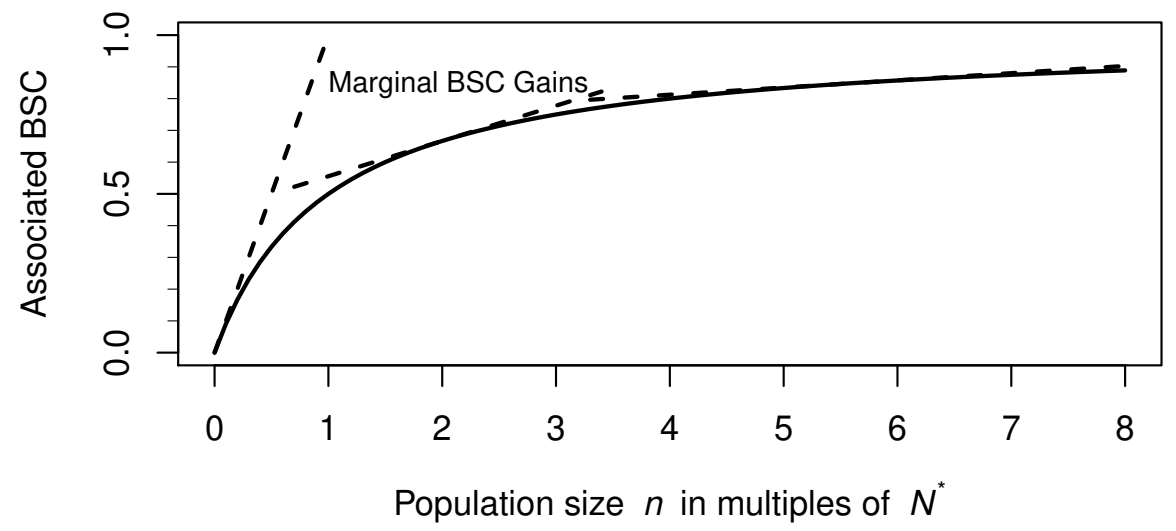

Figure 1: Accumulation of BSC when rebuilding a species at the brink of extinction. The solid line gives the BSC associated with this species, Eq. (14). As illustrated by the dashed lines, BSC gained by increasing the species' population by a single individual are largest when it is closest to extinction. However, to achieve full BSC, the species' population must be lifted well above $N^{*}$, the population size below which random birth and death events cause large population fluctuations.

that the global sum of available BSC is limited to $S$. In fact, market incentives to rebuild the population of $i$ are highest at the lowest population size (Fig. 1).

On the second point, note that market participants that sustain a smaller population of a species $i$ on

their land benefit more from increasing this population than those holding a larger population. The reason is that the latter incur a higher penalty from reducing the BSC of their current holding by increasing $N_{i}$ in the denominator Eq. 10p. Hence BSC disincentivise dominant holdings of species. We therefore expect that market misalignment due to the difference between conditions Eq. (11) and (13) will be harmless in most cases, implying optimal resource allocation as explained above. 


\section{The role of $\mathrm{BSC}$ in biodiversity conservation}

To illustrate the role BSC can play in biodiversity conservation and its limitations, we consider a hypothetical use case. Assume that Organisation X has committed to a net gain in BSC on the land it holds. Conversion of large areas of low productivity agricultural land into solar farms by $\mathrm{X}$ is estimated to reduce the BSC for plants on this land by a total of 0.1 units. To compensate this biodiversity impact, $\mathrm{X}$ works with a conservation NGO, such as the Tree Conservation Fund, to acquire an area of land suitable for the reintroduction of Chinese watermelon trees (Artocarpus nanchuanensis), which are critically endangered, with perhaps only 100 individuals remaining in the wild. Over 1000 saplings of A. nanchuanensis are planted in the area acquired by X. Assume that, after about five years, when the trees mature, over 200 saplings survive. The majority of mature individuals of $A$. nanchuanensis are now on the land owned by $\mathrm{X}$, thus contributing over 0.5 BSC to X's holdings and clearly compensating for the loss of plant BSC from construction of the solar farms. The Tree Conservation Fund estimates the total cost of such measures to be around USD 250,000 38 , which suggests an opening price for a BSC market of around USD 500,000 per species.

Critics might argue that a low-cost, small-scale conservation project cannot compensate the large-scale biodiversity loss due to solar farm construction. Yet, X's use of BSC was as intended by the design of the metric. Capital was directed to a conservation cause where it made a substantial difference at moderate cost. A. nanchuanensis was saved from extinction. Overall, global LPI for plants has increased as a result of X's action. Other early adopters would find similar opportunities to acquire BSC at low cost. As the market for BSC matures, organisations committed to net biodiversity gain would seek more efficient schemes to raise BSC (e.g. by restoring entire ecosystems), thus further improving resource allocation to conservation.

To address the concern noted above, consider, for example, the Mean Species Abundances (MSA) metric, originally called the Biodiversity Intactness Index 28 , a well-established metric which is based on similar data. MSA is defined as the average over all native species in the area of interest and over space of the ratio of 
a species' population to what it would be in an undisturbed ecosystem (capped to be $\leq 1$ ). Assuming the area of the solar farms is much larger than the area of the A. nanchuanensis sanctuary, the combined MSA for solar farms and sanctuary would most likely significantly decrease in the scenario above. To achieve net MSA gain, restoration or rewilding of an area comparable to that of the solar farms would likely be required.

Thus, MSA puts more emphasis on the size of the area, contrasting BSC's emphasis on species conservation. Georgina Mace $^{29}$, in a commentary on MSA, defended this property by arguing that (A) the diversity amongst species as "Irreplaceable, unique units with combinations of traits from long and independent evolution" with "Intrinsic value" is different from "the components of biodiversity that underlie key functions and benefits of an ecosystem", i.e., what we now call ecosystem services; (B) the latter, so the premise, is more important than the former; and (C) MSA's strength lies in capturing this ecosystem service provision. While we agree with Steps A and C of this argument, we argue that the choice made in Step B is not required for a principled approach to conservation.

Building on the international consensus about sustainable development documented in the Brundtland Report ${ }^{39}$, one can formulate a general principle for the sustainable use of ecosystems:

Sustainable use of ecosystems means use [i] to meet the needs of the present [ii] without compromising the ability of future generations to meet their own needs.

Clause ii sets constraints to the implementation of Clause i. Since we cannot know the needs and preferences of future generations, Clause ii effectively constrains the impacts of current uses on ecosystems to those that cannot be undone by the next generation $\underline{40}$ extinctions must be avoided ${ }^{41}$. It calls for recovery of $\mathrm{LPI}_{\text {reg }}$, which can be achieved, e.g., through net BSC gain policies 42 .

There is no easy answer to how to implement Clause [i] under this constraint. Needs and preferences differ between societies ${ }^{40}$. Yet, as a general rule, provision of ecosystem services should be considered, and MSA quantifies these in a first approximation 29 . Accounts of biodiversity impact should therefore capture both aspects: contributions to species conservation (e.g. via BSC) and contributions to conservation of 
ecosystem functioning and services (e.g. via MSA) — and these should not be confounded.

We have demonstrated that Biodiversity Stewardship Credits have properties suitable for use in biodiversity-related disclosures in business and financial contexts. The metric can also support voluntary or legislated no-net-loss policies. In all cases, we recommend its use in conjunction with metrics for ecosystem services or natural capital. Many of the attractive properties of BSC reflect that this metric is strictly science based, mathematically linked to the LPI and the species conservation objective. Data requirements of BSC are similar to those of existing comparable metrics. Pilot studies are now underway to develop suitable protocols for application of BSC in practice. 


\section{Box 2 Example of BSC calculation}

Schoolchildren at Northgate High School and Sixth Form in Ipswich, UK, participating in the Big Schools' Birdwatch organised by the Royal Society for the Protection of Birds (RSPB), counted the birds listed below in the school's Wildlife and Wellbeing Garden on 11 December 2020. Taking these counts at face value (despite some methodological limitations) and combining them with published global abundance estimates ${ }^{30}$, we calculate (table) that the garden holds around $1.3 \times 10^{-6}$ species worth of Bird-BSC. More than half of BSC come from only two species (pie chart): black headed gulls that were abundant in the garden; and blue tits, due to their relatively low global abundance. To demonstrate that we are justified in disregarding $N^{*}$ in these calculations, consider dunnock, the globally rarest species in the sample. From the worst-case literature estimates ${ }^{3132} v_{\mathrm{e}}=0.017 \mathrm{yr}^{-1}$ and $v_{\mathrm{d}}=0.5 \mathrm{yr}^{-1}$, we get $N^{*} \approx 29$, negligible by a wide margin compared to the estimated global abundance $N \approx 9,000,000$.

\begin{tabular}{|c|c|c|c|c|c|}
\hline RSPB common name & $n$ & Scientific name & Glob. abund. $N$ & bsc $=n / N\left(\times 10^{6}\right)$ & Contribution \\
\hline Black headed gull & 34 & Chroicocephalus ridibundus & 78878122 & 0.4310 & $32.7 \%$ \\
\hline Blue tit & 4 & Cyanistes caeruleus & 17115823 & 0.2337 & $17.7 \%$ \\
\hline Long tailed tit & 3 & Aegithalos caudatus & 18889928 & 0.1588 & $12.1 \%$ \\
\hline Magpie & 5 & Pica pica & 33377656 & 0.1498 & $11.4 \%$ \\
\hline Dunnock & 1 & Prunella modularis & 9347093 & 0.1070 & $8.1 \%$ \\
\hline Pied wagtail & 19 & Motacilla alba & 353854467 & 0.0537 & $4.1 \%$ \\
\hline Wren & 1 & Troglodytes troglodytes & 18682476 & 0.0535 & $4.1 \%$ \\
\hline Robin & 1 & Erithacus rubecula & 21261776 & 0.0470 & $3.6 \%$ \\
\hline Greenfinch & 1 & Chloris chloris & 24909193 & 0.0401 & $3.0 \%$ \\
\hline Carrion crow & 1 & Corvus corone & 44906864 & 0.0223 & $1.7 \%$ \\
\hline Woodpigeon & 4 & Columba palumbus & 334511213 & 0.0120 & $0.9 \%$ \\
\hline Blackbird & 3 & Turdus merula & 354433630 & 0.0085 & $0.6 \%$ \\
\hline \multirow[t]{2}{*}{ Sum } & & & & 1.3174 & $100.0 \%$ \\
\hline & & & $\begin{array}{l}\text { - Black he } \\
\text { - Blue tit } \\
\text { - Long tail } \\
\text { - Magpie } \\
\text { - Dunnock } \\
\text { - Pied wac } \\
\text { - Wren } \\
\text { - Robin } \\
\text { - Greenfin } \\
\text { - Carrion } \\
\text { - Woodpig } \\
\text { - Blackbir }\end{array}$ & $\begin{array}{l}\text { ded gull } \\
\text { d tit } \\
\text { ail } \\
\text { ow } \\
\text { on }\end{array}$ & \\
\hline
\end{tabular}

ihttps://community.rspb.org.uk/getinvolved/learning/b/learning-blog/posts/ big-schools-birdwatch-at-northgate-high-school-and-sixth-form 


\section{S1 Numerical test of our model for extinction risk}

Here we demonstrate that Eq. (6) provides a good estimate of mean species lifetime despite making use of the diffusion (continuum) approximation and disregarding the decline of the strength of fluctuations near $x=0$.

For this we simulated an asexual population with non-overlapping generations as

$$
\begin{aligned}
\lambda(t) & \sim \exp \left(v_{\mathrm{e}}^{1 / 2} \xi(t)\right), \\
N(t+1) & \sim \operatorname{Poisson}(\lambda(t) N(t)),
\end{aligned}
$$

with $\xi(t)$ denoting a standard normal random number and Poisson $(\mu)$ a Poisson-distributed random number with mean $\mu$. The model implies $v_{\mathrm{d}}=1$.

We set $v_{\mathrm{e}}=(0.15)^{2}$ and evaluated 26 values for the initial population size $N(0)=N_{0}$, spaced equally on the $\log$ axis from 1 to $10^{5}$, except for rounding to the nearest integer. For each $N_{0}$, the probability of species survival $(N(T)>0)$ until time $T=10^{4}$ was estimated from $10^{4}$ replicates.

As shown in Fig. S1 1 , extinction probability increases linearly with $\log \left(N_{0}\right)$ only for large $N_{0}$. By contrast, $x_{0}=\log \left(1+N_{0} / N^{*}\right)$, with $N^{*}=v_{\mathrm{e}} / v_{\mathrm{e}}=44.4$, is linearly related to extinction probability for all $N_{0}$ considered (because probabilities are $\leq 1$ by definition, the relationship would break down for even larger $N_{0}$ ). The slope of the relation is as predicted by Eq. (6). 

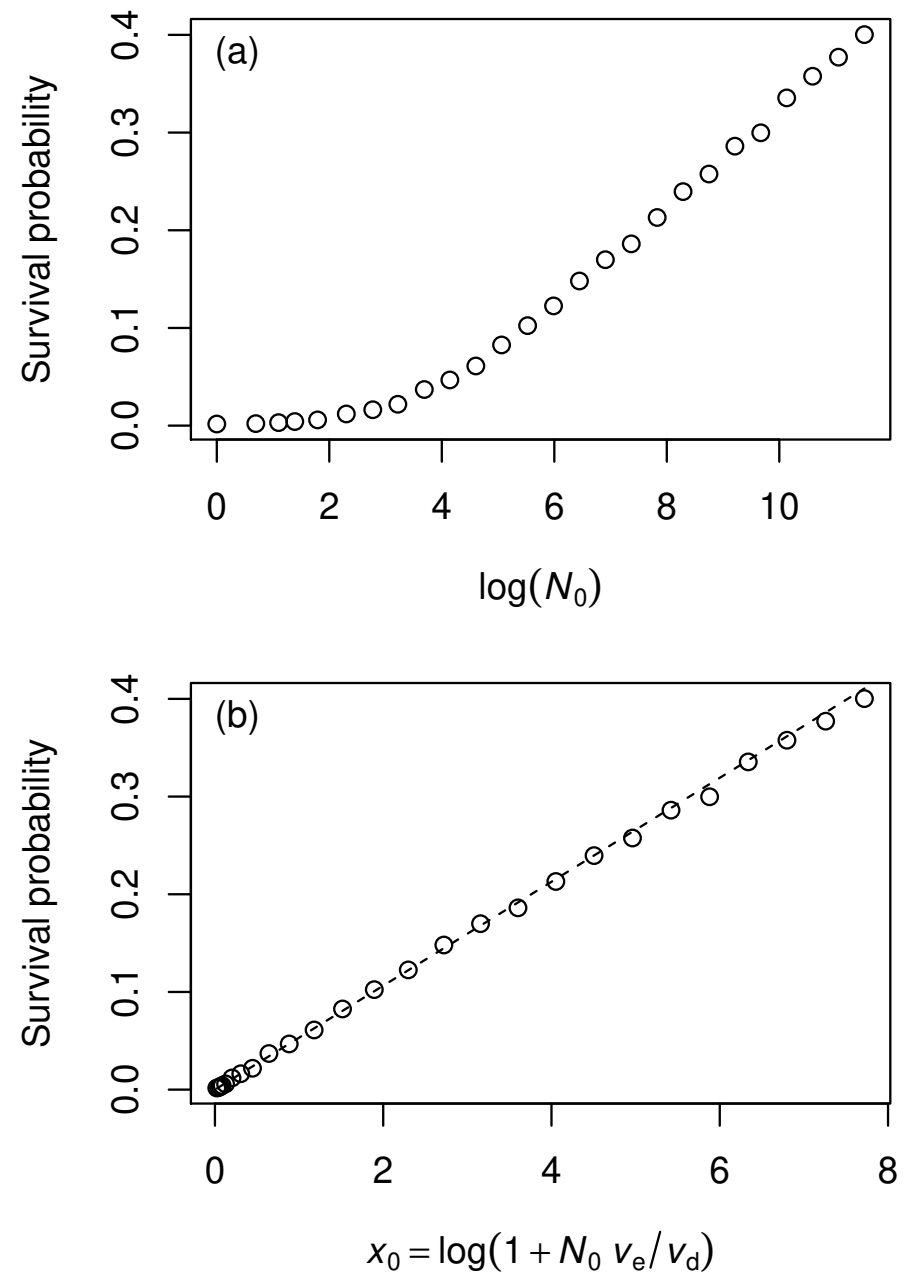

Figure S1: Demonstration that $x_{0}$ predicts expected species lifetime better than $\log \left(N_{0}\right)$. Points are simulation results for the individual-based model Eq. (S1) with $v_{\mathrm{e}}=(0.15)^{2}$ over $T=10^{4}$ time steps. The dashed line is the probability of survival as approximated by Eq. (6). Panels (a) and (b) represent the same data on different horizontal scales. 


\section{S2 Comparison of BSC with the Species Threat Abatement and Recovery (STAR) metric}

To understand its relation of BSC to the Species Threat Abatement and Recovery (STAR) metric ${ }^{44}$, let us first note that, if the current area of the habitat suitable to a species, $\mathrm{AoH}^{[45}$, is known and one assumes, for simplicity, even distributions over the habitat, then the ratio $n_{i} /\left(N_{i}^{*}+N_{i}\right)$ entering the definition of BSC, Eq. (10), can, for $N_{i} \gg N_{i}^{*}$, be approximated by the proportion $P_{i}$ of species $i$ 's habitat contained in the area to be assessed. Hence

$$
\mathrm{BSC} \approx \sum_{i}^{S} P_{i} .
$$

The definition of STAR has a similar structure, but with two additional factors in the sum. Firstly, the numeric red-list category $W_{i}$ of species $i$, an integer ranging from 0 (Least Concern) to 4 (Critically Endangered). Secondly, an index $C_{i, t}$ quantifying either the relative contribution of threat $t$ to the extinction risk of that species, or the contribution threat abatement could make to $i$ 's recovery. Hence

$$
\operatorname{STAR}_{t}=\sum_{i}^{S} P_{i} W_{i} C_{i, t} .
$$

The factor $C_{i, t}$ apportioning impacts over threats adds semantics to STAR that BSC do not carry (though the idea might be transferable). The technical difference between STAR and BSC thus lies mainly in the threat-level factor $W_{i}$. We have argued above that extinction risk is already captured by $n_{i} /\left(N_{i}^{*}+N_{i}\right)$, approximated by $P_{i}$, suggesting that the $W_{i}$ factor might duplicate this information. Indeed, the extent of occurrence of a species, closely related to $\mathrm{AoH}^{45}$, is a central red-listing criterion. Besides, inclusion of the factor $W_{i}$ implies choices that may not have an obvious justifications: for example, why not use instead a factor $W_{i}^{3}$ or $W_{i}^{1 / 3}$ ? However, since the range of values that $W_{i}$ can attain is rather narrow, the overall effect 


\section{${ }_{303}$ References}

[1] Diab, A. \& Adams, G. M. ESG Assets May Hit $\$ 53$ Trillion by 2025, a Third of Global AUM. Bloomberg Professional Services (2021). https://www.bloomberg.com/professional/blog/ esg-assets-may-hit-53-trillion-by-2025-a-third-of-global-aum/.

[2] Addison, P. F. E., Bull, J. W. \& Milner-Gulland, E. J. Using Conservation Science to Advance Corporate Biodiversity Accountability. Conserv. Biol. 33, 307-318 (2019).

[3] Nauman, B. ESG Investors Wake up to Biodiversity Risk. Financial Times (2020). https://www.ft. com/content/100f0c5b-83c5-4e9a-8ad0-89af2ea4a758.

[4] Smith, T. et al. Biodiversity Means Business: Reframing Global Biodiversity Goals for the Private Sector. Conserv. Lett. 13, e12690 (2020).

[5] TNFD. Proposed Technical Scope-Recommendations for the TNFD. Tech. rep. (2021). https:// tnfd.global/wp-content/uploads/2021/07/TNFD-\%E2\%80\%93-Technical-Scope-3.pdf.

[6] Soto-Navarro, C. A. et al. Towards a Multidimensional Biodiversity Index for National Application. Nat Sustain 4, 933-942 (2021).

[7] Bull, J. W., Suttle, K. B., Gordon, A., Singh, N. J. \& Milner-Gulland, E. J. Biodiversity Offsets in Theory and Practice. Oryx 47, 369-380 (2013).

[8] Jones, P. Biodiversity Net Gain and Planning. Town Ctry. Plan. 401-405 (2021). 
[9] TNFD. Nature in Scope - A Summary of the Proposed Scope, Governance, Work Plan, Communication and Resourcing Plan of the TNFD. Tech. rep., Taskforce on Nature-related Financial Disclosures (2021). http://www.incp.org.co/Site/publicaciones/info/archivos/TNFD-Nature-in-Scope.pdf

[10] UNEP-WCMC. Technical Workshop on Aligning Biodiversity Measurement Approaches for Business. Tech. rep. (2019).

[11] WWF. Living Planet Report 2020 - Bending the Curve of Biodiversity Loss. Tech. rep., World Wildlife Fund, Gland, Switzerland (2020).

[12] McRae, L., Deinet, S. \& Freeman, R. The Diversity-Weighted Living Planet Index: Controlling for Taxonomic Bias in a Global Biodiversity Indicator. PLOS ONE 12, e0169156 (2017).

[13] Leung, B. et al. Clustered versus Catastrophic Global Vertebrate Declines. Nature 588, 267-271 (2020).

[14] Santini, L. et al. Assessing the Suitability of Diversity Metrics to Detect Biodiversity Change. Biological Conservation 213, 341-350 (2017).

[15] Rossberg, A. G. \& Farnsworth, K. D. Simplification of Structured Population Dynamics in Complex Ecological Communities. Theor. Ecol. 4, 449-465 (2011).

[16] Collen, B. et al. Monitoring Change in Vertebrate Abundance: The Living Planet Index. Conserv. Biol. 23, 317-327 (2009).

[17] Kalyuzhny, M. et al. Niche versus Neutrality: A Dynamical Analysis. The American Naturalist 184, 439-446 (2014).

[18] Kessler, D., Suweis, S., Formentin, M. \& Shnerb, N. M. Neutral Dynamics with Environmental Noise: Age-size Statistics and Species Lifetimes. Phys. Rev. E 92, 022722 (2015). 
[19] Melbourne, B. A. Stochasticity, Demographic. In Encyclopedia of Theoretical Ecology, chap. Encyclopedia of Theoretical Ecology, 706-712 (University of California Press, 2012).

[20] Pechenik, L. \& Levine, H. Interfacial Velocity Corrections Due to Multiplicative Noise. Phys. Rev. E 59, 3893-3900 (1999).

[21] Dornic, I., Chaté, H. \& Muñoz, M. A. Integration of Langevin Equations with Multiplicative Noise and the Viability of Field Theories for Absorbing Phase Transitions. Phys. Rev. Lett. 94, 100601 (2005).

[22] Kessler, D. A., Ner, Z. \& Sander, L. M. Front Propagation: Precursors, Cutoffs, and Structural Stability. Phys. Rev. E 58, 107-114 (1998).

[23] Pande, J., Tsubery, Y. \& Shnerb, N. M. Quantifying Invasibility (2021).

[24] Gardiner, C. W. Handbook of Stochastic Methods (Springer, Berlin, 1990), second edn.

[25] Abramowitz, M. \& Stegun, I. A. (eds.). Handbook of Mathematical Functions (Dover, New York, 1972).

[26] O’Sullivan, J. D., Terry, J. C. D., Wilson, R. \& Rossberg, A. G. Predictors of the Long-Term Conservation Value of Sites in a Mechanistic Metacommunity Model. bioRxiv 2021.07.09.451808 (2021).

[27] Weissgerber, M., Roturier, S., Julliard, R. \& Guillet, F. Biodiversity Offsetting: Certainty of the Net Loss but Uncertainty of the Net Gain. Biological Conservation 237, 200-208 (2019).

[28] Scholes, R. J. \& Biggs, R. A Biodiversity Intactness Index. Nature 434, 45-49 (2005).

[29] Mace, G. M. An Index of Intactness. Nature 434, 32-33 (2005).

[30] Callaghan, C. T., Nakagawa, S. \& Cornwell, W. K. Global Abundance Estimates for 9,700 Bird Species. PNAS 118 (2021). 
[31] Sæther, B.-E., Grøtan, V., Engen, S., Noble, D. G. \& Freckleton, R. P. Critical Parameters for Predicting Population Fluctuations of Some British Passerines. J. Anim. Ecol. 78, 1063-1075 (2009).

[32] Sæther, B.-E. et al. Life-History Variation Predicts the Effects of Demographic Stochasticity on Avian Population Dynamics. Am. Nat. 164, 793-802 (2004).

[33] IUCN. IUCN Red List Categories and Criteria, Version 3.1, Second Edition (IUCN, 2012). https: //portals.iucn.org/library/node/10315.

[34] Enquist, B. et al. The Commonness of Rarity: Global and Future Distribution of Rarity across Land Plants. Science Advances 5, eaaz0414 (2019).

[35] Engen, S., Lande, R., Saether, B.-E. \& Dobson, F. S. Reproductive Value and the Stochastic Demography of Age-Structured Populations. Am. Nat. 174, 795-804 (2009).

[36] Sæther, B.-E. et al. How Life History Influences Population Dynamics in Fluctuating Environments. Am. Nat. 182, 743-759 (2013).

[37] Chiang, A. C. \& Wainwright, K. Fundamental Methods of Mathematical Economics (McGraw Hill, 2005), fourth edn.

[38] Tree Conservation Fund. Artocarpus Nanchuanensis - BGCI's Tree Conservation Fund. https://www. treeconservationfund.org/species/artocarpus-nanchuanensis/ (2021).

[39] World Commission on Environment and Development. Our Common Future. Oxford Paperbacks (Oxford University Press, Oxford ; New York, 1987).

[40] Rossberg, A. G. et al. Quantitative Criteria for Choosing Targets and Indicators for Sustainable Use of Ecosystems. Ecological Indicators 72, 215-224 (2017).

[41] Rounsevell, M. D. A. et al. A Biodiversity Target Based on Species Extinctions. Science (2020). 
[42] Bull, J. W. et al. Net Positive Outcomes for Nature. Nat Ecol Evol 4, 4-7 (2020).

[43] Czúcz, B. et al. Where Concepts Meet the Real World: A Systematic Review of Ecosystem Service Indicators and Their Classification Using CICES. Ecosystem Services 29, 145-157 (2018).

[44] Mair, L. et al. A Metric for Spatially Explicit Contributions to Science-Based Species Targets. Nat Ecol Evol 5, 836-844 (2021).

[45] Brooks, T. M. et al. Measuring Terrestrial Area of Habitat (AOH) and Its Utility for the IUCN Red List. Trends in Ecology 85 Evolution 34, 977-986 (2019). 